Lymphoscintigraphic imaging study for quantitative evaluation of a small field of view (SFOV) gamma camera

This content has been downloaded from IOPscience. Please scroll down to see the full text. 2015 JINST 10 P07011

(http://iopscience.iop.org/1748-0221/10/07/P07011)

View the table of contents for this issue, or go to the journal homepage for more

Download details:

IP Address: 143.210.120.158

This content was downloaded on 19/08/2015 at 16:09

Please note that terms and conditions apply. 


\title{
Lymphoscintigraphic imaging study for quantitative evaluation of a small field of view (SFOV) gamma camera
}

\author{
M.S. Alqahtani, ${ }^{a, b, 1}$ J.E. Lees, ${ }^{a}$ S.L. Bugby, ${ }^{a}$ L.K. Jambi ${ }^{a, c}$ and A.C. Perkins ${ }^{d}$ \\ ${ }^{a}$ Space Research Centre, Department of Physics and Astronomy, University of Leicester, \\ University Road, Leicester, LE1 7RH, U.K. \\ ${ }^{b}$ Radiological Sciences Department, College of Applied Medical Sciences, King Khalid University, \\ Postcode: 3665, Zip code 61481, Abha, Kingdom of Saudi Arabia \\ ${ }^{c}$ Radiological Sciences Department, College of Applied Medical Sciences, King Saud University, \\ Postcode: 10219, Zip code 11433, Riyadh, Kingdom of Saudi Arabia \\ ${ }^{d}$ Radiological and Imaging Sciences Department, School of Medicine, University of Nottingham, \\ Derby Road, Nottingham, NG7 2UH, U.K.
}

E-mail: msma7@leicester.ac.uk

AbstRACT: The Hybrid Compact Gamma Camera (HCGC) is a portable optical-gamma hybrid imager designed for intraoperative medical imaging, particularly for sentinel lymph node biopsy procedures. To investigate the capability of the HCGC in lymphatic system imaging, two lymphoscintigraphic phantoms have been designed and constructed. These phantoms allowed quantitative assessment and evaluation of the HCGC for lymphatic vessel (LV) and sentinel lymph node (SLN) detection. Fused optical and gamma images showed good alignment of the two modalities allowing localisation of activity within the LV and the SLN. At an imaging distance of $10 \mathrm{~cm}$, the spatial resolution of the HCGC during the detection process of the simulated LV was not degraded at a separation of more than $1.5 \mathrm{~cm}$ (variation $<5 \%$ ) from the injection site (IS). Even in the presence of the IS the targeted LV was detectable with an acquisition time of less than 2 minutes. The HCGC could detect SLNs containing different radioactivity concentrations (ranging between 1:20 to 1:100 SLN to IS activity ratios) and under various scattering thicknesses (ranging between $5 \mathrm{~mm}$ to $30 \mathrm{~mm}$ ) with high contrast-to-noise ratio (CNR) values (ranging between 11.6 and 110.8). The HCGC can detect the simulated SLNs at various IS to SLN distances, different IS to SLN activity ratios and through varied scattering medium thicknesses. The HCGC provided an accurate physical localisation of radiopharmaceutical uptake in the simulated SLN. These characteristics of the HCGC reflect its suitability for utilisation in lymphatic vessel drainage imaging and SLN imaging in patients in different critical clinical situations such as interventional and surgical procedures.

KEYWORDS: Gamma camera, SPECT, PET PET/CT, coronary CT angiography (CTA); Scintigraphy and whole-body imaging; Multi-modality systems; Intra-operative probes

\footnotetext{
${ }^{1}$ Corresponding author.
} 


\section{Contents}

1 Introduction 1

2 Hybrid Compact Gamma Camera design 2

3 Materials and methods $\quad 3$

3.1 Phantom construction 3

3.1.1 Lymphatic vessel phantom design and activity simulation 3

3.1.2 Melanoma phantom design and activity simulation 3

3.2 Injection site (IS), lymphatic vessel (LV) and sentinel lymph node (SLN) activity simulation challenge

3.2.1 Lymphatic vessel activity simulation

3.2.2 Sentinel lymph node (SLN) activity simulation 5

3.3 Imaging procedure 5

3.4 Measured parameters 6

4 Results and discussion 6

4.1 Characterisation of HCGC performance with a lymphatic vessel phantom 6

4.1.1 Performance comparison between LFOV and SFOV gamma cameras 7

4.1.2 Gamma and hybrid imaging with a lymphatic vessel phantom $\quad 8$

4.2 Characterisation of HCGC performance with a melanoma phantom 8

4.2.1 Detectability of the sentinel lymph node (SLN) 9

4.2.2 Influence of relative injection site position on SLN detectability 11

$\begin{array}{ll}\text { 4.2.3 Influence of radioactivity concentration on LV and SLN appearance } & 14\end{array}$

5 Conclusion 14

\section{Introduction}

The lymphatic system performs the chief function of monitoring tissue fluid balance through emptying interstitial pores and conserving protein concentrations in order to preserve colloid osmotic pressure [1]. Various pathologies are developed if the lymphatic system is unable to function normally. The most common is lymphedema, in which tissues are damaged beyond repair as a result of interruption of lymphatic transport [2]. The lymphatic system has also attracted attention for its part in tumour metastasis and in dermal drug delivery [3-6]. The role of the lymphatic system in the spread of diseases and therapy processes has necessitated swift progress in the diagnostic imaging tools that are capable of quantifying lymphatic system function via providing functional imaging of lymphatic contractions and lymph flow towards lymph nodes. 


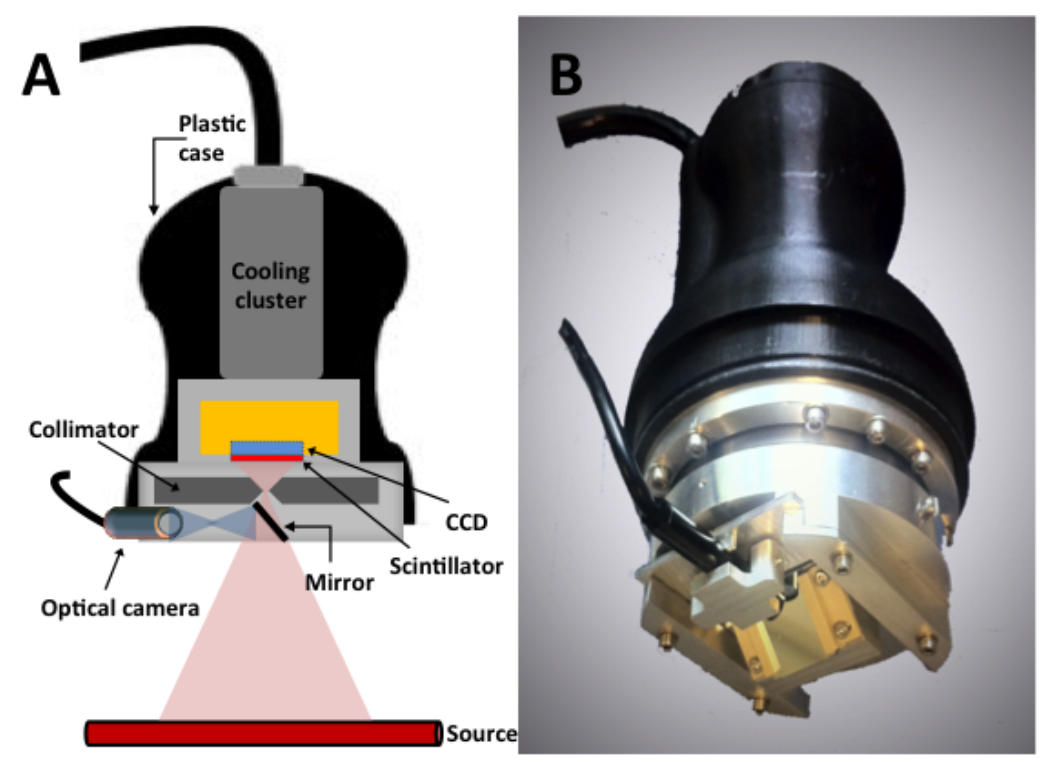

Figure 1. (A) Schematic view of the HCGC. A thin (3 mm thickness) first surface mirror at 45 degrees and an optical camera outside the direct line of sight of the pinhole provides co-aligned optical imaging. (B) An image showing the HCGC in the proposed configuration.

Functional imaging of the lymphatic system in nuclear medicine is known as lymphoscintigraphy. A common procedure involves isolating the sentinel lymph node (SLN), the first node that receives lymph drainage from a tumour, performing a biopsy or surgery to identify the stage of cancer and generating a corresponding treatment plan. Lymphoscintigraphy can also locate the blockage position in the lymphatic vessels (LVs), such as lymph flow in an arm or leg, or lymphedema [7-10]. In routine medical practice, lymphoscintigraphy makes use of a conventional large field of view (LFOV) gamma camera to obtain images. These cameras are unavailable for intraoperative imaging so, if required during surgery, regions of high activity are identified using non-imaging gamma probes. The proposed benefits of intraoperative imaging have led to a number of new intraoperative imaging systems currently in development [11, 12].

In this paper, the suitability of a new intraoperative imaging system - the Hybrid Compact Gamma Camera (HCGC) - for lymphoscintigraphy and intraoperative SLN imaging has been investigated. The techniques and phantoms used in this report are applicable to any SFOV gamma imaging system and may be used for quantitative performance comparisons.

\section{Hybrid Compact Gamma Camera design}

The basic components of the HCGC are an e2v CCD97 back illuminated (BI) charge-coupled device (CCD) [13], a caesium iodide scintillator doped with thallium CsI(Tl) coupled to the CCD and a single-pinhole collimator. A reconstruction algorithm interprets the CCD data, providing the position and energy for each individual gamma photon interaction [14]. The magnification of the system depends on the distance from the pinhole collimator to the object under examination.

Figure 1A shows the way that gamma photons from the source pass through the mirror with minimal absorption ( $<3 \%$ for $140 \mathrm{keV}$ photons) and minimal scatter, whereas optical photons are 
reflected by the mirror towards the optical camera. The results of both optical and gamma cameras are captured simultaneously and may be displayed separately or as a combined image. The HCGC is therefore able to provide high spatial resolution multi-modality imaging.

The use of pinhole collimators for the HCGC has two main advantages; it simplifies the design and it increases the possible imaging field of view whereas a parallel-hole collimator would restrict the field of view (FOV) to the image area of the CCD $\sim 8 \mathrm{~mm} \times 8 \mathrm{~mm}$ [14]. The collimators are manufactured from tungsten discs, $6 \mathrm{~mm}$ thick and $45 \mathrm{~mm}$ in diameter. The extrinsic sensitivity using $0.5 \mathrm{~mm}$ diameter pinhole collimator is $214 \mathrm{cps} / \mathrm{MBq}$. Keeping in view the requisites of spatial resolution and sensitivity required in a range of applications, $0.5 \mathrm{~mm}$ or $1.0 \mathrm{~mm}$ pinhole diameters can be utilised, each having an acceptance angle of $60^{\circ}$ [15]. More details about the HCGC characterisation and design have been reported elsewhere $[14,16]$.

\section{Materials and methods}

\subsection{Phantom construction}

To evaluate the ability of HCGC for use in sentinel node lymphoscintigraphy, two lymphoscintigraphic phantoms have been developed and fabricated in-house. These phantoms are constructed from Perspex (methyl methacrylate PMMA) plates. Perspex has been utilised in manufacturing these medical phantoms because of its acceptable degree of similarity with human tissue [17].

\subsubsection{Lymphatic vessel phantom design and activity simulation}

The first phantom, a lymphatic vessel phantom, has been constructed to assess the capability of the HCGC for lymphatic vessel drainage imaging. It consists of a $10 \mathrm{~mm}$ diameter Eppendorf tube containing about $60 \mathrm{MBq}$ of ${ }^{99 \mathrm{~m}} \mathrm{Tc}(0.5 \mathrm{ml})$ imitating the injection site, and a lymphatic vessel simulated by an $80 \mathrm{~mm}$ long capillary tube ( $1.1 \mathrm{~mm}$ internal diameter) filled by approximately $16 \mathrm{MBq}$ of ${ }^{99 \mathrm{~m}} \mathrm{Tc}$ solution $(0.1 \mathrm{ml})$. The capillary tube was placed underneath thicknesses of Perspex ranging from $5 \mathrm{~mm}$ to $30 \mathrm{~mm}$ to simulate the lymphatic vessel at different depths inside the human body with a superficial injection site. This simulated lymphatic vessel (LV) is comparable in size to natural lymphatic vessels; for instance, the diameters of lymphatic vessels in the distal parts of the lower extremities range between $0.8 \mathrm{~mm}$ and $1.5 \mathrm{~mm}$ [18].

\subsubsection{Melanoma phantom design and activity simulation}

The second phantom, a melanoma phantom, is a combination phantom. It simulates the injection site, the lymphatic vessel and SLN of approximately average size [19]. The melanoma phantom was used to evaluate the relationship between activity uptake and the HCGC sensitivity at different depths. The effect of the distance from the injection site to the sentinel lymph node on the image quality was also evaluated utilising this phantom. The configuration of the first phantom has been used as a basis for this phantom; however, two square Perspex plates $(80 \times 80 \mathrm{~mm})$ with $8 \mathrm{~mm}$ thickness have been added. One plate contains a $7 \mathrm{~mm}$ diameter $(6 \mathrm{~mm}$ depth) well to mimic a SLN, and the second plate has a $70 \times 70 \mathrm{~mm}$ square well to simulate the background activity around the SLN. Figure 2 shows an illustration of both lymphoscintigraphic phantom designs utilised in this study. 



Figure 2. (A) A schematic of the lymphatic vessel phantom showing the simulated lymphatic vessel (LV) and the injection site (IS); (B) melanoma phantom configuration showing the simulated sentinel lymph node (SLN), IS, LV and the background well.

Table 1. Summary of simulated activity in the melanoma phantom.

\begin{tabular}{|c|c|c|c|}
\hline SLN to IS ratio & IS activity in $0.5 \mathrm{ml}$ & SLN activity in $0.1 \mathrm{ml}$ & $\begin{array}{c}\text { Background } \\
\text { activity in } 4 \mathrm{ml}\end{array}$ \\
\hline $1: 20$ & \multirow{3}{*}{$15 \mathrm{MBq}$} & $0.75 \mathrm{MBq}$ & $3 \mathrm{MBq}$ \\
\hline $1: 50$ & & $0.3 \mathrm{MBq}$ & $1.2 \mathrm{MBq}$ \\
\hline $1: 100$ & & $0.15 \mathrm{MBq}$ & $0.6 \mathrm{MBq}$ \\
\hline
\end{tabular}

For the melanoma phantom, the simulated injection site (i.e. Eppendorf tube) was filled with $0.5 \mathrm{ml}$ of a ${ }^{99 \mathrm{~m}} \mathrm{Tc}$ solution (15 MBq total) [20]. The SLN was simulated by various activities with SLN to IS ratios ranging between 1:20 to $1: 100$ in $0.1 \mathrm{ml}$ solutions. The simulated activity of the background was one tenth of the SLN activity (i.e. 1:10 BG to SLN ratio). The $80 \mathrm{~mm}$ long lymphatic vessel (capillary tube) contained the same activity in the SLN distributed over approximately $0.1 \mathrm{ml}$ (see table 1$)$.

\subsection{Injection site (IS), lymphatic vessel (LV) and sentinel lymph node (SLN) activity simulation challenge}

\subsubsection{Lymphatic vessel activity simulation}

The lack of clinical data for estimated activity concentrations in LVs in the human body during lymphoscintigraphic procedures and the difficulty in performing these kinds of studies is a result of the nature of lymphatic vessels. They have varying and uncertain diameters and depths; even for a known lymphatic route, the proximal and distal parts can differ in diameter and depth [18]. Moreover, the physiological condition of the examined part specifically and the whole body generally plays a primary role in determining the ratio of the radioactivity which is trapped in the LVs [21, 22]. Many external factors can affect the uptake ratio in the LVs, such as the amount of administrated activity and the time delay between the administration of the activity and the scanning procedure. The scattering effect produced by hot spots (e.g. the IS) close to the LV may also 
influence the ability to detect it. Therefore, the different amounts of activity used to simulate the activity in the LV in both phantoms were selected to determine the detection limit of the HCGC.

\subsubsection{Sentinel lymph node (SLN) activity simulation}

In clinical practice, it is a laborious task to determine the specific ratio of SLN to IS activity since there are many radiocolloids (radiopharmaceuticals) with widely varying molecular diameters, which affect the activity flow and spread. The ratio is also strongly affected by time delays between the initial administration of the radioactivity and the surgical procedure. Furthermore, the SLNs vary extensively in size, shape and the number of lymphatic suppliers, which may also affect the radioactive dose uptake [23]. In addition, many authors endorse stress lymphoscintigraphy for the extremities after the administration of the radioactivity; although this medical practice is not globally employed, it enhances the sensitivity and improves lymphatic flow and the activity uptake in the SLNs, which affects the SLN to IS activity ratio [24-26].

A formalised standardisation of the radioactive material dose to be administered in SLN procedures has not yet been adopted. The suggested and investigated activities vary between $3.7 \mathrm{MBq}$ and $370 \mathrm{MBq}$ depending on the procedure and institution; however, 5 to $30 \mathrm{MBq}$ as the total injected dose for SLN detection scanning is generally accepted and can be considered a sufficient dose for same-day surgical procedures [20].

For this study, activity simulations for melanoma were conducted for SLN to IS activity ratios of 1:20, 1:50 and 1:100. This range was selected to cover the majority of values in studies [27-29], and to investigate the capability of the HCGC in a range of medical scenarios. To improve the simulation, an active background has been added to imitate the uptake in the soft tissues around the SLN. The typical background to SLN activity ratio ranges between 1:10 and 1:20 [30]. This variation depends upon the type and the amount of the injected activity, and delay time between the dose administration and surgery may also affect this ratio. In this study, 1:10 background activity to SLN activity ratio was used.

\subsection{Imaging procedure}

The HCGC was used to image both phantoms. The lymphatic vessel phantom was also imaged using a standard/conventional large field of view (LFOV) gamma camera (Nucline ${ }^{\mathrm{TM}} \mathrm{X}-\mathrm{Ring}-\mathrm{R}$ (HR), -C) equipped with a low-energy high-resolution (LEHR) collimator (extrinsic sensitivity with LEHR collimator $144 \mathrm{cps} / \mathrm{MBq}$ ) [31]. For the HCGC, the $0.5 \mathrm{~mm}$ and $1 \mathrm{~mm}$ pinhole collimators were both used in this study. The SLN and LV in both phantoms were placed beneath different thicknesses of scattering medium, ranging between $5 \mathrm{~mm}$ and $30 \mathrm{~mm}$. In the melanoma phantom, nuclear scans were obtained over varied distances between the IS and the SLN. The IS to SLN centre-to-centre distance was varied between $10 \mathrm{~mm}$ and $50 \mathrm{~mm}$ in $10 \mathrm{~mm}$ intervals. A metallic frame with a clamp held the HCGC perpendicular to the phantom's surface. The distance between the collimator and the Perspex surface was $100 \mathrm{~mm}$. The acquisition time for both phantoms was between $\sim 100 \mathrm{~s}$ and $\sim 500 \mathrm{~s}$.

After the acquisition procedure was performed, specific steps are initiated to enhance the image quality produced by the HCGC, and improve the accuracy of the analysis. The main step is "Blob" detection technique, whereby a Gaussian distribution is fitted to each light 'splash' produced from the scintillator. Two types of Blob detection modes can be produced; Cumulative and 
Centre-Point detection modes. Centre-Point image takes the peak of the Gaussian fit and plots this only whereas the Cumulative image takes the whole Gaussian fit and plots the whole spread of each splash. A detailed explanation of Blob detection technique is explained elsewhere [14, 32].

In this study, all the data produced by the HCGC and used to produce graphs are taken from Centre-Point mode images with different acquisition periods, and all gamma and hybrid images presented in this study are enhanced utilising Cumulative detection mode. These images were also processed using Image-J software Gaussian Blur filter (Gaussian sigma $=1$ pixel) [33]. It is important to mention that the view and the acquisition technique for Centre-Point mode are comparable to that produced by standard LFOV gamma cameras.

\subsection{Measured parameters}

For the lymphatic vessel phantom, count profiles were obtained with a fixed collimator to surface distance $(100 \mathrm{~mm})$. The target object (i.e. the lymphatic vessel) was positioned underneath different Perspex thicknesses (figure 2A). The HCGC was held perpendicular to the surface of the Perspex. The full width at half maximum (FWHM) of the count profiles was recorded.

For the melanoma phantom, the SLN was placed beneath a variety of scattering medium thicknesses (figure 2B). The HCGC was fixed $10 \mathrm{~mm}$ away from the surface of the scattering medium. The contrast to noise ratio (CNR) is calculated for the sentinel lymph node at different depths (ranging from $5 \mathrm{~mm}$ to $30 \mathrm{~mm}$ ), fixed distance (i.e. $20 \mathrm{~mm}$ ) from the injection site and various SLN to IS ratios (i.e. 1:20, 1:50 and 1:100). CNR measurements should be conducted for a small field of view gamma camera to advise the operator if a hot spot (e.g. lesion or SLN) can definitely be assessed as detectable or not. Rose (1973) gives an approximation that in order to be detectable an object's CNR must exceed 3-5 [34]. The CNR calculation formula utilised in this study is as follows $[35,36]$

$$
\mathrm{CNR}=\left[\frac{\left(\mathrm{N}_{1}-\mathrm{N}_{\mathrm{bg}}\right)}{\sigma_{\mathrm{bg}}}\right] .
$$

Where $\mathrm{N}_{l}$ corresponds to the mean counts of a lesion area (i.e. SLN) and $\mathrm{N}_{\mathrm{bg}}$ is the average background count level. $\sigma_{\mathrm{bg}}$ is the background counts' standard deviation. The Image-J and Origin 9.1 [37] programmes have been utilised for analysing and plotting purposes. A circular region of interest corresponding to the lesion area was defined based on the actual simulated SLN's size and shape (i.e. $7 \mathrm{~mm}$ diameter); also, a background region of interest of identical size was chosen.

\section{Results and discussion}

\subsection{Characterisation of HCGC performance with a lymphatic vessel phantom}

Lymphatic mapping for surgical or biopsy procedures is based on the idea that the lymphatic fluid drains from a primary malignant tumour site to a specific regional lymph node. Moreover, a common definition for the SLN concept is that provided by Morton et al., which defines a sentinel lymph node as any lymph node on the afferent (i.e. towards the node) drainage pathway from the primary tumour [38]. This definition reflects the importance of the lymphatic vessel detection, and its integral part in detecting and determining the SLN. Therefore, the lymphatic vessel phantom has been constructed to assess the HCGC capability in LV detection. 


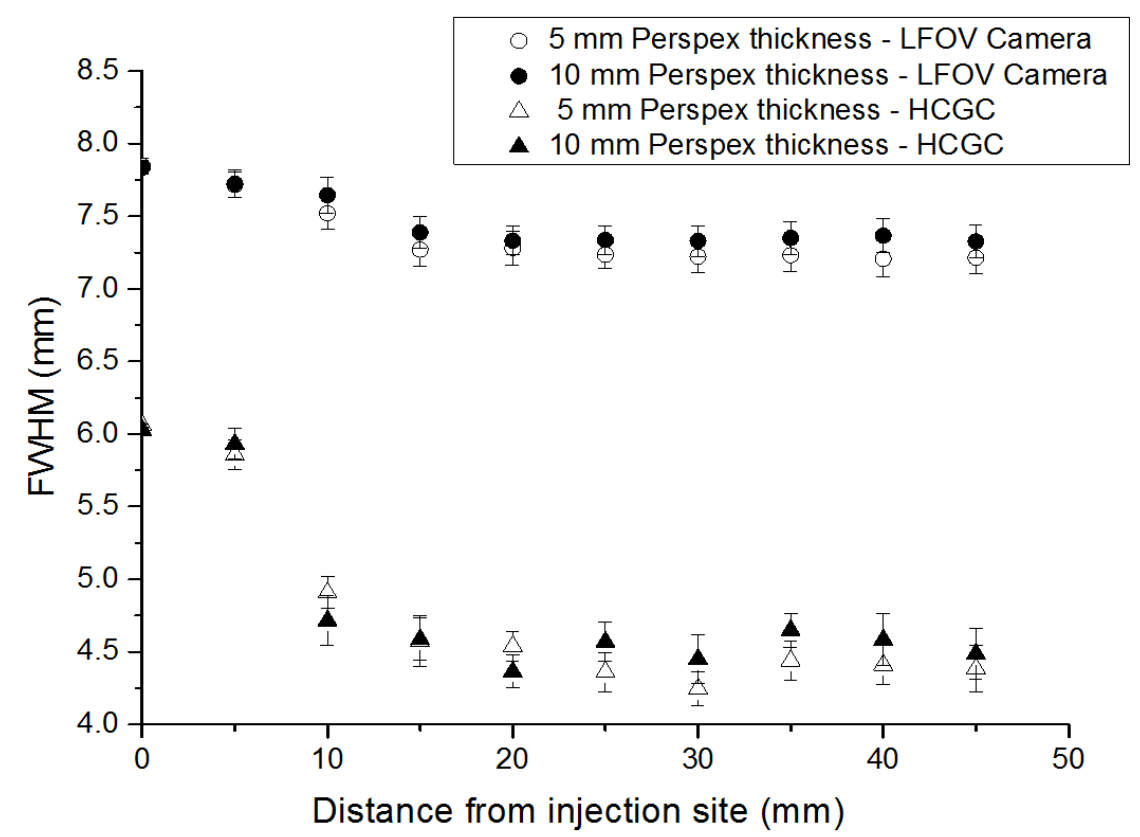

Figure 3. Comparison of the full width at half maximum (FWHM) of the lymphatic vessel phantom as a function of distance from the centre of the IS for the HCGC and the standard LFOV gamma camera at an acquisition time of $\sim 500 \mathrm{~s}$. Circles: FWHM of count profiles produced by the standard LFOV gamma camera with 5 and $10 \mathrm{~mm}$ of the scattering medium. Triangles: FWHM of count profiles acquired by the HCGC with 5 and $10 \mathrm{~mm}$ of the scattering medium. The collimator to phantom distance for both cameras was $100 \mathrm{~mm}$. The distance $0 \mathrm{~mm}$ represents the centre of the IS.

\subsubsection{Performance comparison between LFOV and SFOV gamma cameras}

Figure 3 shows the relationship between the measured FWHM of the simulated LV at different distances from the IS for both the standard LFOV gamma camera and the HCGC. The measured spatial resolution was significantly better (i.e. smaller FWHM) for the HCGC than the standard LFOV gamma camera, independent of separation or depth of scattering material. The FWHM of the modelled lymphatic vessel remains at a steady level (variation $<5 \%$ ) when the distance from the centre of the IS was more than $\sim 15 \mathrm{~mm}$, reflecting the fundamental spatial resolution of each camera (measured at $100 \mathrm{~mm}$ from the phantom).

In figure 4 , at a fixed acquisition time of $\sim 500 \mathrm{~s}$, the steady increase in the full width at half maximum (FWHM) shows the degradation in spatial resolution with an increase of scattering medium thickness. The poorest spatial resolution recorded by the HCGC in this experiment is $6.3 \mathrm{~mm}$ at $130 \mathrm{~mm}$ collimator to source distance and $30 \mathrm{~mm}$ Perspex thickness. Nevertheless, these results are better than those produced by the standard LFOV gamma camera ranging between $7.1 \mathrm{~mm}$ and $8.1 \mathrm{~mm}$ underneath $5 \mathrm{~mm}$ to $30 \mathrm{~mm}$ of scattering material.

It may be noticed that the spatial resolution of the HCGC system degrades slightly faster with increasing thicknesses of scattering medium due to the nature of pinhole collimation. However, for clinically relevant scenarios the influence of the degradation in spatial resolution between the two gamma cameras remained relatively small given the limited depths of the LVs inside the human body in most cases. 


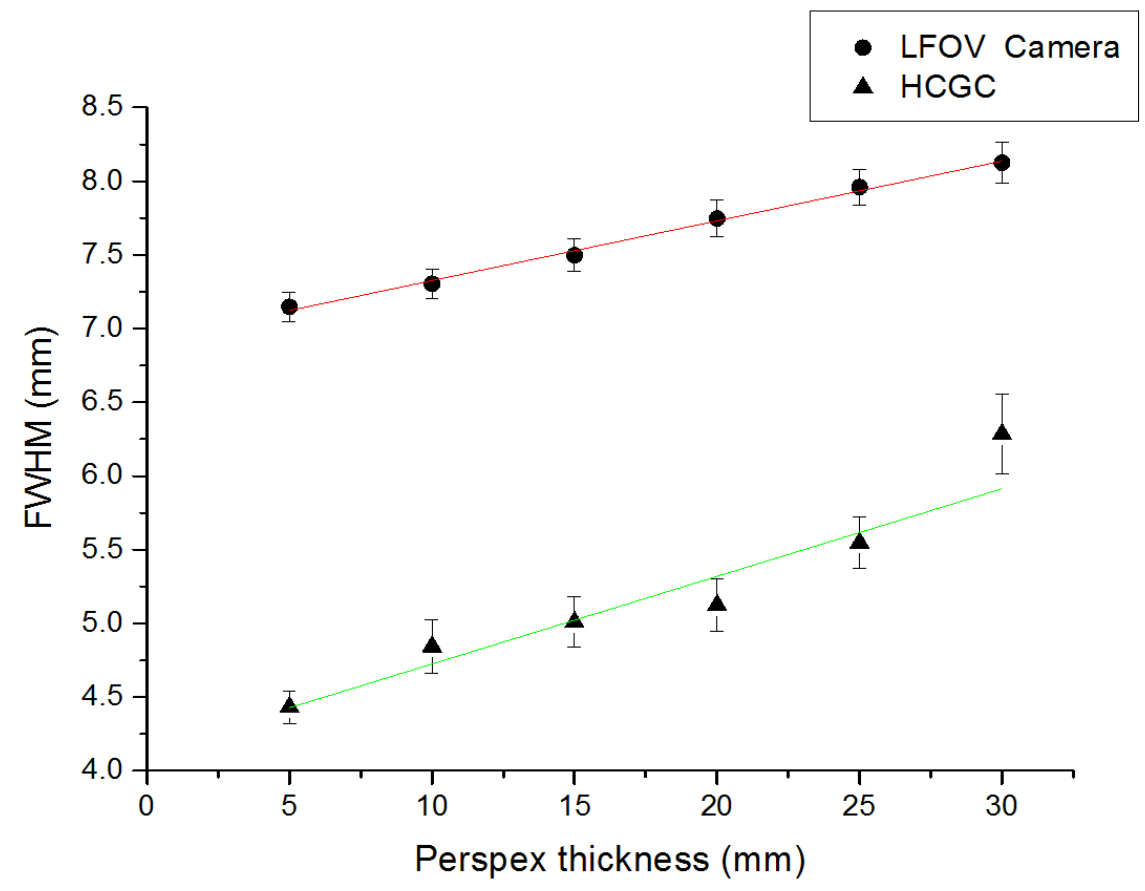

Figure 4. Full width at half maximum (FWHM) of the lymphatic vessel phantom as measured by a conventional LFOV gamma camera and the HCGC. The simulated IS was not used during the acquisition of the gamma images analysed to produce this graph.

\subsubsection{Gamma and hybrid imaging with a lymphatic vessel phantom}

The concept of hybrid imaging is proposed as a way of improving the HCGC capability of activity localisation during nuclear imaging procedures. The clinical advantage of presenting gamma and optical images simultaneously would be appreciated, mainly in the intraoperative situation, because of the valuable anatomical and functional information provided. Hybrid optical and gamma images of the simulated lymphatic vessel are produced by the HCGC, as shown in figure 5. They show good co-alignment of the optical set with the HCGC. This co-aligned configuration of the HCGC allows an accurate localisation of hot spots within the field of view. The ability to recognise the texture and the shape of abnormal tissues (e.g. lesions) by viewing the fused image, providing functional and anatomical information simultaneously, may improve the HCGC detection sensitivity over non-hybrid SFOV gamma systems.

In figure 6, a dual injection site has been simulated with two Eppendorf tubes. The simulated dual injection site is located within the field of view (FOV). Even in the presence of the high-activity dual injection site $(\sim 80 \mathrm{MBq})$ the targeted lymphatic vessel can be imaged within an acquisition time of $\sim 100 \mathrm{~s}$. Increasing the acquisition time to approximately $500 \mathrm{~s}$ further enhances the gamma image contrast (figure 6B).

\subsection{Characterisation of HCGC performance with a melanoma phantom}

The use of nuclear substances for detection purposes during SLN biopsy procedures in patients with melanoma is a well-recognised medical practice [39]. These procedures use LFOV gamma cameras 



Figure 5. Optical (A), gamma (B) and hybrid (C) images for the simulated lymphatic vessel. The middle capillary tube was filled with ${ }^{99 \mathrm{~m}} \mathrm{Tc}$ mixed with blue dye; the upper and lower tubes were filled with green dye only. The gamma image was taken over $\sim 500 \mathrm{~s}$ with the LVs at a $5 \mathrm{~mm}$ Perspex depth.
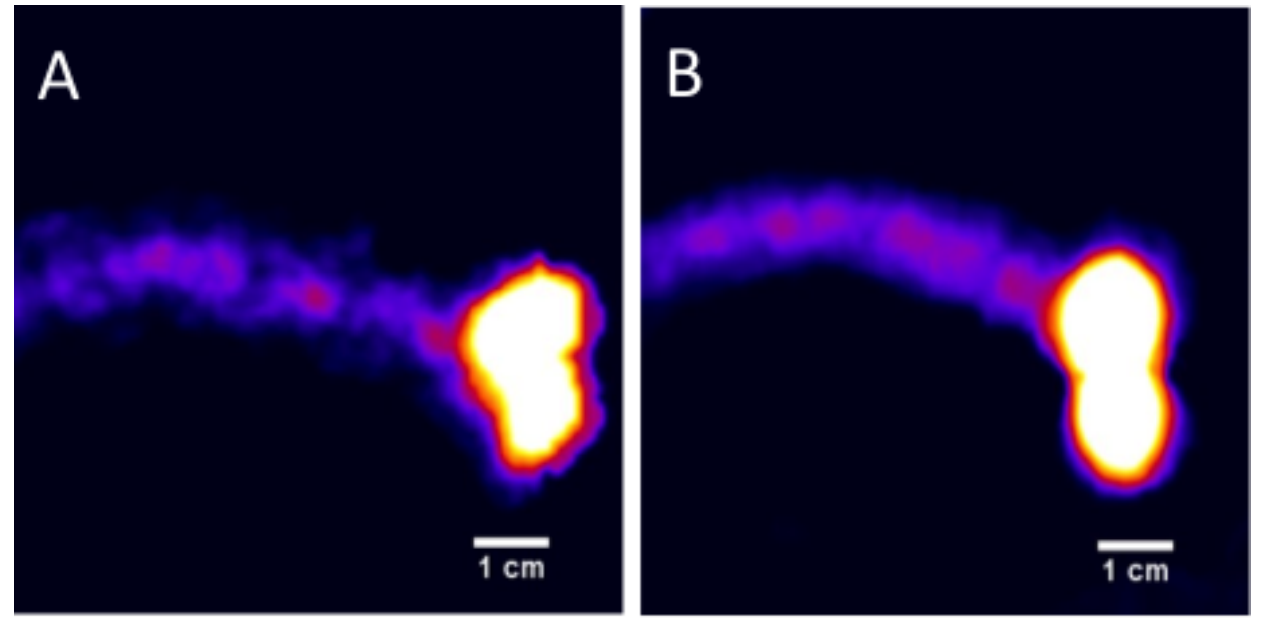

Figure 6. Gamma images showing the lymphatic vessel phantom, including the dual IS, for two different acquisition periods; image (A) has an acquisition time of $\sim 100 \mathrm{~s}$ and image (B) of $\sim 500 \mathrm{~s}$. The LV was placed underneath $5 \mathrm{~mm}$ of Perspex, with the dual IS placed on the surface of the scattering material.

pre-operatively and gamma probes intraoperatively. However, this study introduces the HCGC to the field of SLN detection in melanoma cases and evaluates its capability in SLN detection in different clinical scenarios. The melanoma phantom described in section 3.1, figure $2 \mathrm{~B}$ is used to simulate the SLN at different positions and varied concentrations of radioactivity.

\subsubsection{Detectability of the sentinel lymph node (SLN)}

The detectability of a SLN by a small field of view (SFOV) gamma camera is associated with the CNR. In figure 7, the SLN to IS activity ratios were 1:20, 1:50 and 1:100, and analysis was performed on gamma images produced by the HCGC (fitted with $0.5 \mathrm{~mm}$ diameter pinhole collimator) with $\sim 100 \mathrm{~s}$ acquisition time. Within this short acquisition time, the HCGC is able to detect SLNs containing different concentrations of radioactivity with high CNR values. For instance, the worst CNR value recorded is 11.6 at $30 \mathrm{~mm}$ depth with 1:100 SLN to IS activity ratio; however, the SLN 




Figure 7. Relationship between CNR and varying thicknesses of scattering material (i.e. Perspex plates) for the SLN at $20 \mathrm{~mm}$ away from the IS, and $100 \mathrm{~mm}$ from the HCGC collimator to the surface of the melanoma phantom. The IS activity was $15 \mathrm{MBq}$. The dotted line at $\mathrm{CNR}=2.5$ shows a calculated threshold value based on the Rose criterion of lesion detectability.

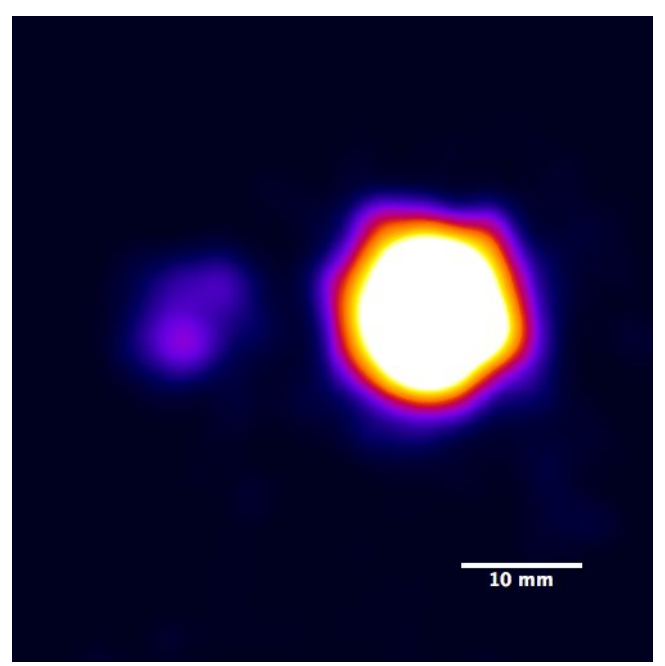

Figure 8. Gamma image for the SLN at $20 \mathrm{~mm}$ IS to SLN distance (centre to centre); the SLN were placed beneath $30 \mathrm{~mm}$ of Perspex, and the SLN to IS activity ratio was 1:100.

is clearly detected as seen in figure 8 . This value is higher than the threshold value (i.e. 3-5) that stated in the Rose criterion [34]. Consequently, the HCGC's capability to detect SLNs at various scattering thicknesses and with different concentrations of activity reflects the usefulness of the HCGC in critical clinical situations (e.g. surgical or interventional procedures).

It can be seen in figure 7 that changes in radioactivity uptake effectively influence the HCGC's ability to detect the SLN; however, this effect becomes less pronounced with increasing thick- 


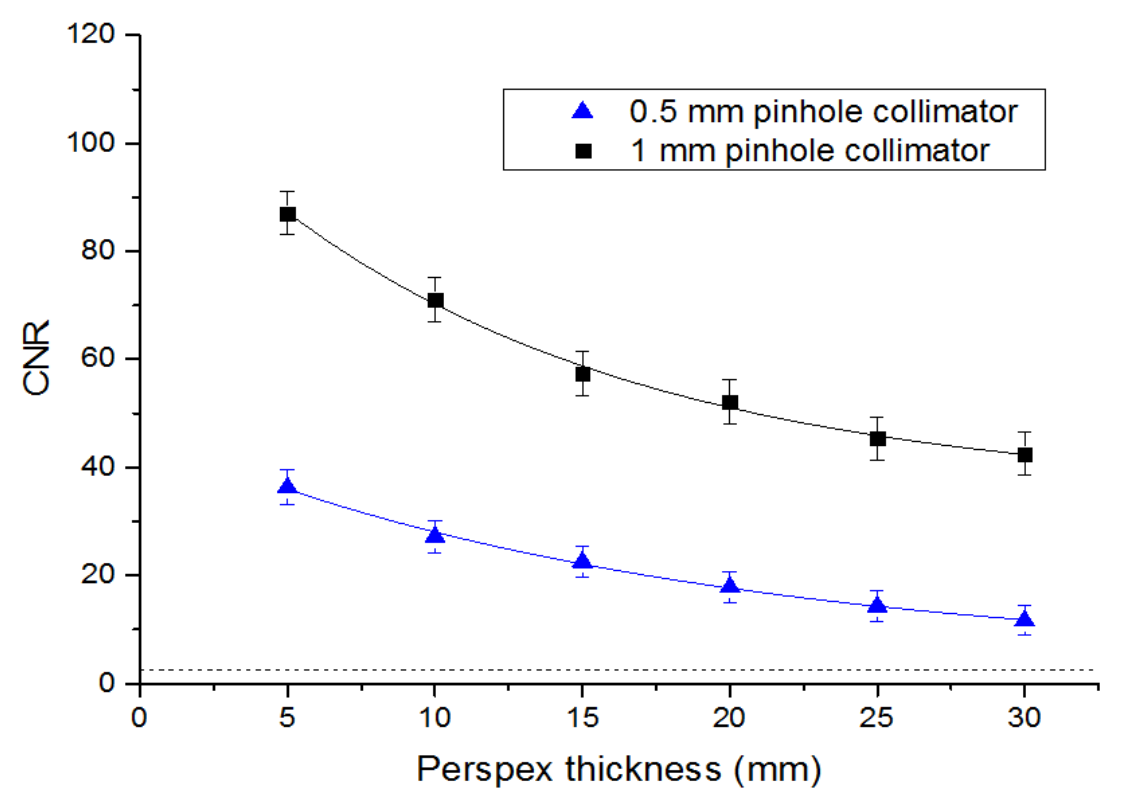

Figure 9. Relationship between CNR and varying thicknesses of Perspex for the SLN at $20 \mathrm{~mm}$ away from the IS, and $100 \mathrm{~mm}$ from the HCGC collimator to the surface of the melanoma phantom. $0.5 \mathrm{~mm}$ and $1 \mathrm{~mm}$ pinhole collimators were used. The IS activity was $15 \mathrm{MBq}$. The dotted line at CNR $=2.5$ shows a calculated threshold value based on the Rose criterion of lesion detectability.

nesses of scattering material. Therefore, for deep SLNs in some clinical cases, applying compression during the functional imaging procedure (e.g. by using compressing pads) will improve the detectability while maintaining a large field of view.

The effect of scattering material on the HCGC detectability can be also minimised by shortening the collimator to surface distance, which will however reduce the field of view, but enhance the system spatial resolution, improve sensitivity, reduce scattering radiation and improve detectability. Furthermore, the choice of a wider pinhole collimator (i.e. $1 \mathrm{~mm}$ diameter) will improve the detectability when the separation between the IS and the SLN is larger compared to the change in the spatial resolution; however, the system spatial resolution of the HCGC will be affected. A comparison of the detectability between a $0.5 \mathrm{~mm}$ and $1 \mathrm{~mm}$ diameter pinhole collimators has been made (see figure 9). The poorest CNR value recorded for the $1 \mathrm{~mm}$ diameter pinhole collimator is 42.5 at $30 \mathrm{~mm}$ depth with 1:100 SLN to IS radioactivity ratio. This value is significantly higher than the value recorded by using $0.5 \mathrm{~mm}$ diameter pinhole collimator at the same condition (i.e. same depth and activity concentration),

In addition, acquisition time greatly affects detectability. To detect SLNs with small size, low activity uptake or at depth, longer acquisition times would be recommended. These adaptations would make the HCGC a suitable tool for functional imaging under different clinical conditions.

\subsubsection{Influence of relative injection site position on SLN detectability}

In figure 10, the IS and the SLN were separated by a centre-to-centre distance of $10 \mathrm{~mm}$, i.e. a $1.5 \mathrm{~mm}$ gap separation when the size of the IS and the SLN are taken into account. Using $0.5 \mathrm{~mm}$ diameter pinhole collimator, the HCGC is able to clearly resolve the SLN at the closest point to the 


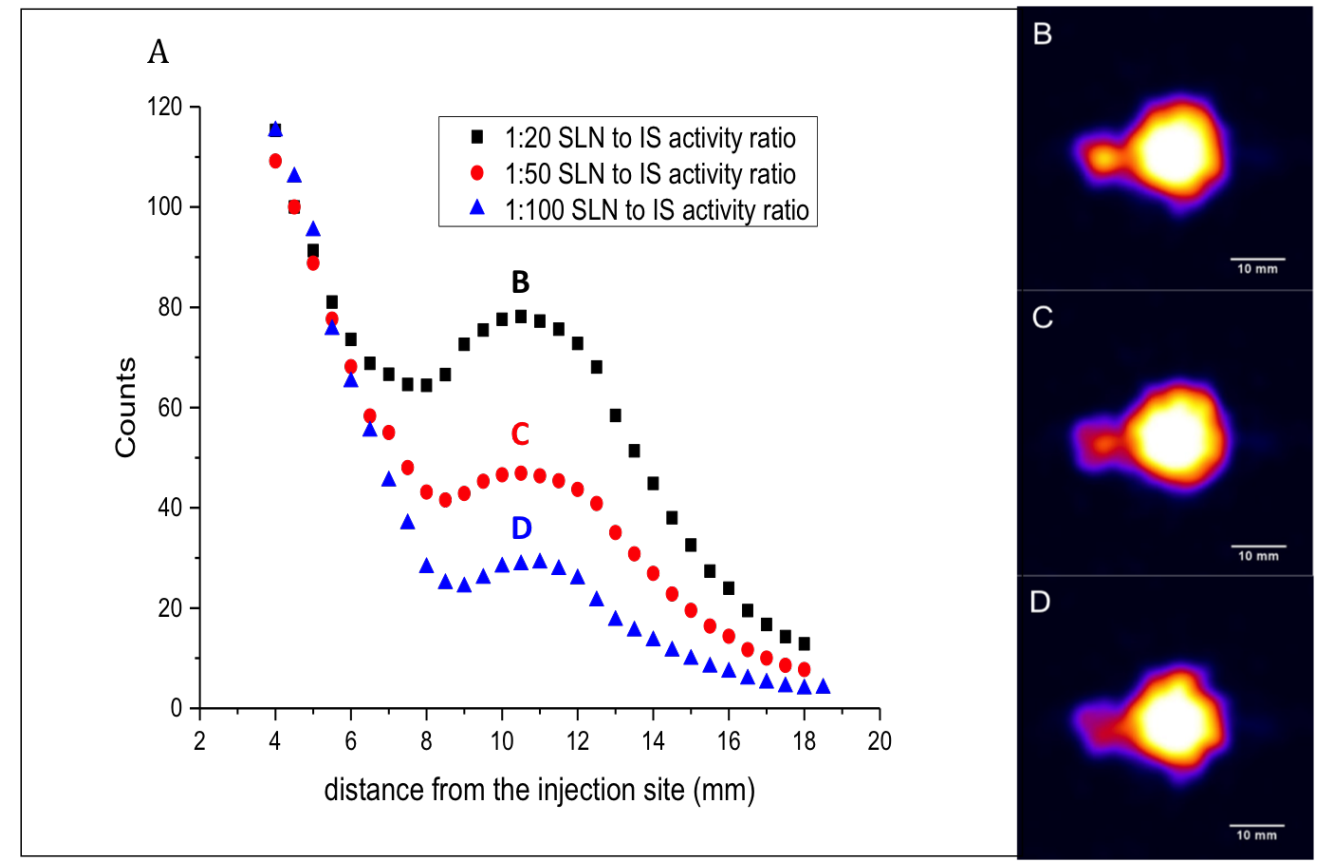

Figure 10. (A) Plot of count profiles for the closest simulated SLN to the IS underneath $10 \mathrm{~mm}$ of the scattering medium. (B, C and D) Gamma images of the SLN at 1:20, 1:50 and 1:100 SLN to IS activity ratios respectively ( $10 \mathrm{~mm}$ IS to SLN distance). The acquisition time for the images analysed in this graph was $\sim 200 \mathrm{~s}$. The IS activity was $15 \mathrm{MBq}$.

IS. As previously discussed, the difference in the concentration of radioactivity noticeably affects the HCGC ability to detect lymph nodes. Nevertheless, even with the 1:100 SLN to IS activity ratio the HCGC is still capable of resolving the peak of the SLN count profile curve within a $\sim 200 \mathrm{~s}$ acquisition period. According to the SLN concept discussed above the ability of the HCGC to detect the closest SLN to the IS with good spatial resolution would improve the diagnostic process.

Figure 11 represents the gamma images obtained by the HCGC equipped with $0.5 \mathrm{~mm}$ diameter pinhole collimator for the SLN at $10 \mathrm{~mm}$ Perspex depth and various IS to SLN distances ranging from $20 \mathrm{~mm}$ to $50 \mathrm{~mm}$. These gamma images were analysed to produce the count profile curves for simulated SLN with an acquisition time of $\sim 200 \mathrm{~s}$ as shown in figure 12. The apparent SLN count level decreases as the SLN is located stepwise further away from the IS. This behaviour reflects the effect of IS activity on neighbouring anatomical structures. As the amount of radioactivity is increased in the IS, the scattering caused by this activity is increased. However, the HCGC is able to resolve counts peaks (i.e. SLNs) at different IS to SLN distances effectively.

In addition, administration of high amounts of radioactivity in the IS would increase the uptake (i.e. the detectability) in targeted SLNs far from the IS such as inguinal SLNs, which may be useful for functional imaging during metastasis biopsy procedures. However, neighbouring SLNs (i.e. close to the IS) may be flooded by scattering of the IS radioactivity. Therefore, for nuclear radioguided SLN biopsy in patients with suspected malignant cutaneous melanoma, the activity of the administrated dose ranging between 7 and $20 \mathrm{MBq}$ in small volumes (i.e. 0.05 to $0.2 \mathrm{ml}$ based on the skin thickness of the examined anatomical area) [30, 40]. This practice will improve the HCGC's ability to detect neighbouring SLNs and avoid any chance of collapsing LVs. 


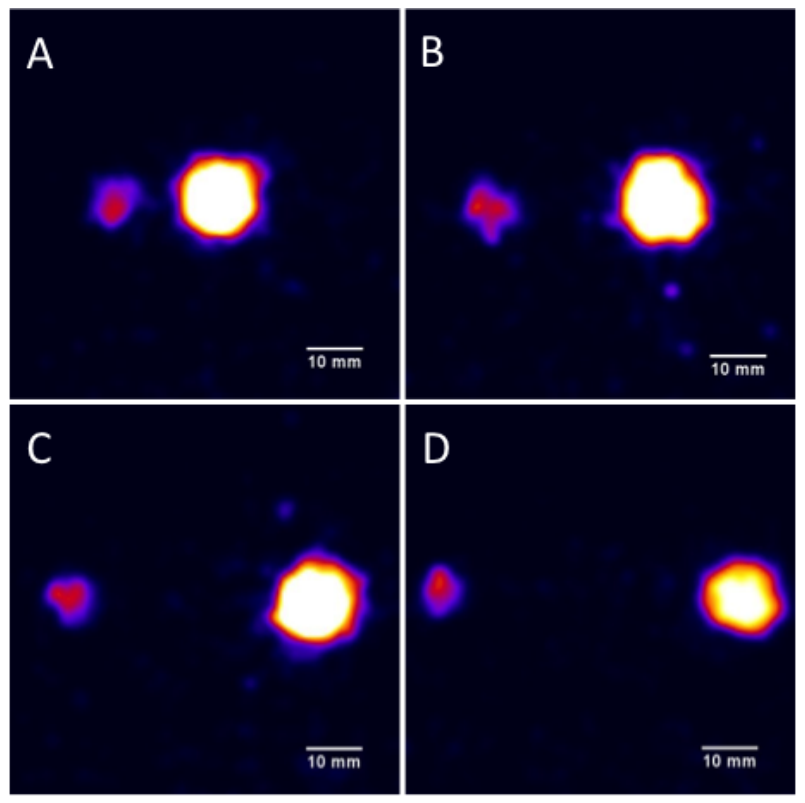

Figure 11. Gamma images for the SLN at different IS to SLN distance (centre to centre); (A) 20 mm away from the IS, (B) $30 \mathrm{~mm}$, (C) $40 \mathrm{~mm}$ and (D) $50 \mathrm{~mm}$. The SLN and LV were placed beneath $10 \mathrm{~mm}$ of Perspex, and the SLN to IS activity ratio was 1:100.

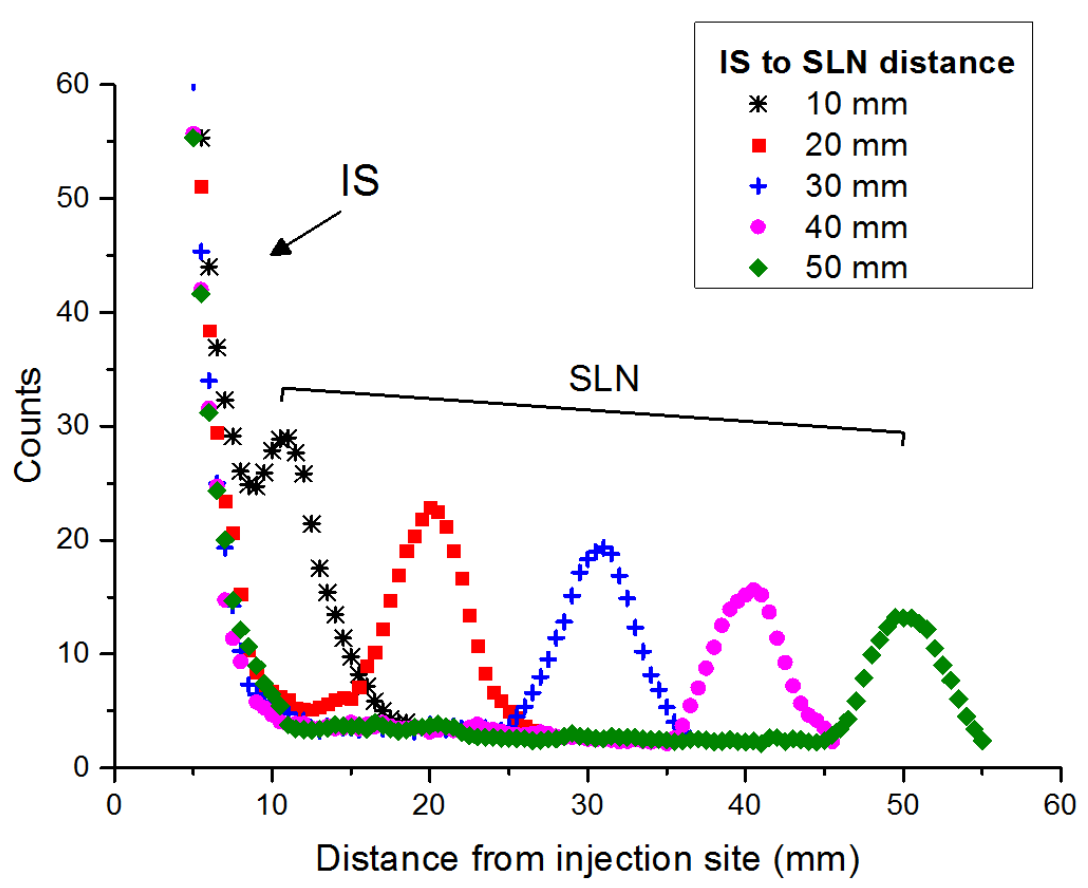

Figure 12. Count profile curves of the melanoma phantom obtained at different IS to SLN distances. The SLN to IS ratio was 1:100 and the SLN was located underneath $10 \mathrm{~mm}$ of Perspex. Peaks due to IS and SLNs are labelled. The simulated IS activity was $15 \mathrm{MBq}$. 

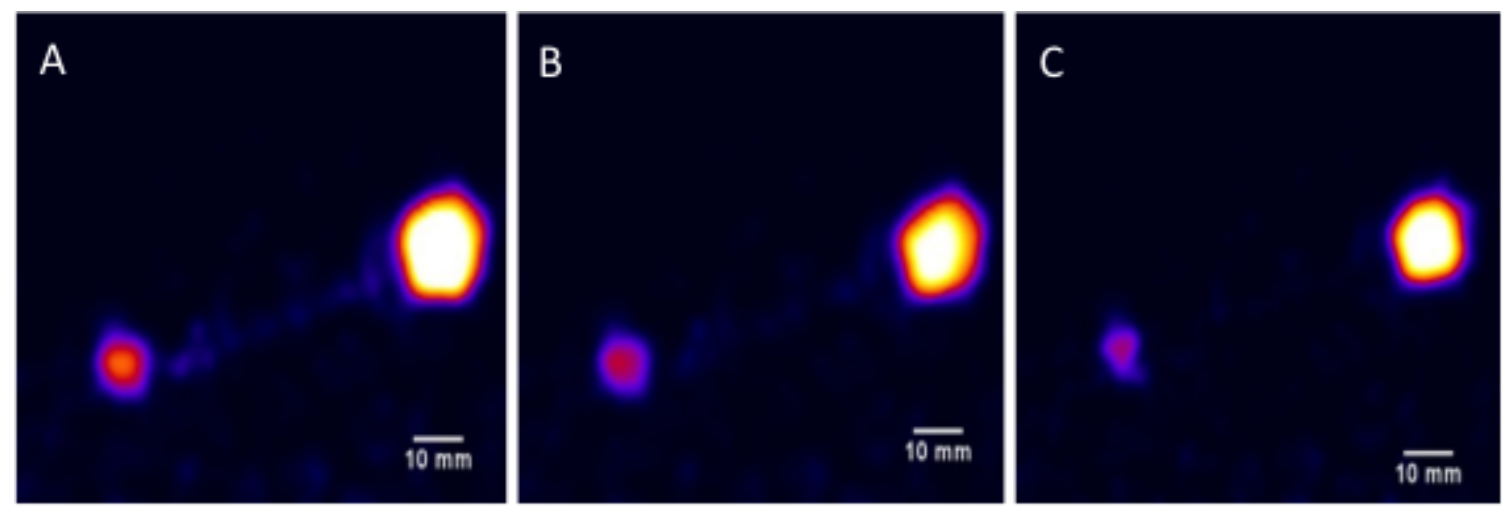

Figure 13. Gamma images of SLN and LV placed underneath $30 \mathrm{~mm}$ of Perspex and the superficial IS; the SLN to IS activity ratio is 1:20 in (A), 1:50 in (B) and 1:100 in (C). The SLN to IS distance is $50 \mathrm{~mm}$. The IS activity was $15 \mathrm{MBq}$.

\subsubsection{Influence of radioactivity concentration on LV and SLN appearance}

In figure 13, the same gamma imaging procedure is performed on a specific configuration of the melanoma phantom. The simulated SLN and LV were placed underneath $30 \mathrm{~mm}$ of Perspex, and the SLN to IS distance was set at $50 \mathrm{~mm}$. However, the SLN and LV to IS activity ratio was varied between 1:20 and 1:100. The acquisition time during this imaging process was $\sim 200 \mathrm{~s}$.

The SLN is clearly detected for all investigated phantom configurations (i.e. at IS to SLN distances ranging between $10 \mathrm{~mm}$ and $50 \mathrm{~mm}$; and thicknesses of Perspex ranging between $5 \mathrm{~mm}$ and $30 \mathrm{~mm}$ ) and for all simulated SLN to IS activity ratios for melanoma detection. The simulated $\mathrm{LV}$ of $80 \mathrm{~mm}$ length is detectable with 1:20 LV to IS activity ratio (i.e. a $0.75 \mathrm{MBq}$ dose). However, the LV was not clearly imaged with activity ratios of $1: 50$ and $1: 100(0.3 \mathrm{MBq}$ and $0.15 \mathrm{MBq})$ at large depths (figure 13B and C). It is important to clarify that the activity ratios used with the simulated LV in this study are not estimated based on clinical data, and that the selection of the simulated LV to IS activity ratios for the LV in the melanoma phantom was made to determine the limit of detection with the HCGC.

The observed changes in the SLN size (figure 13) in the gamma images follow changes in the SLN activity concentration; i.e. the SLN appears smaller with lower radioactivity concentration. However, the optical camera would improve the accuracy of SLN localisation with various activity concentrations; i.e. by inspecting the hybrid image, even if activity is strongly accumulated on a specific side of the SLN, the discrimination of the edge of the SLN and its texture from the surrounding tissues would be easier.

\section{Conclusion}

The lymphatic system is currently the focus of interest because of its significant role in tumour metastasis. A simulation technique and two lymphoscintigraphic phantoms have been illustrated and utilised to validate the capability of the Hybrid Compact Gamma Camera (HCGC) in lymphatic system imaging. The implementation of this quantitative assessment enables the practical performance of the HCGC to be evaluated for lymphatic vessel (LV) and sentinel lymph node 
(SLN) localisation. The HCGC performance as characterised by this experiment clarifies that it is appropriate for lymphatic vessel drainage imaging and SLN imaging in patients with melanoma. The anatomical view provided by the optical camera improves physical localisation for the radioactivity trapped in LVs and SLNs. A high spatial resolution and high contrast to noise ratios have been recorded in this study. The HCGC detected the simulated LV with a spatial resolution ranging between 4.4 and $6.3 \mathrm{~mm}$ at $100 \mathrm{~mm}$ collimator to surface distance, and it was able to detect the simulated SLN that containing various radioactivity concentrations with high contrast-to-noise ratios (CNRs) values ranging between 11.6 and 110.8, underneath different scattering thicknesses ranging between $5 \mathrm{~mm}$ to $30 \mathrm{~mm}$. This HCGC capability to detect the simulated LV and SLN reflects its suitability for use in critical clinical situations such as interventional and surgical procedures. Further assessment will be performed with the purpose of testing the HCGC in a surgical theatre setting.

\section{Acknowledgments}

The authors would like to thank Helen Hill and David Monk, the Leicester Royal Infirmary, for their technical support. Also, the authors would like to thank William McKnight, Space Research Centre, University of Leicester, and A H Ng, Radiological and Imaging Sciences, Medical School, University of Nottingham for their advice and assistance. M.S. Alqahtani and L.K. Jambi have been financially supported by King Khalid University and King Saud University, Ministry of Education, Kingdom of Saudi Arabia. S.L. Bugby acknowledges support through an STFC studentship.

\section{References}

[1] R.M. Dongaonkar, G.A. Laine, R.H. Stewart and C.M. Quick, Balance point characterization of interstitial fluid volume regulation, Am. J. Physiol. Regul. Integr. Comp. Physiol. 297 (2009) R6.

[2] K. Radhakrishnan and S.G. Rockson, The clinical spectrum of lymphatic disease, Ann. New York Acad. Sci. 1131 (2008) 155.

[3] T. Karpanen and K. Alitalo, Molecular biology and pathology of lymphangiogenesis, Annu. Rev. Pathol. 3 (2008) 367.

[4] A.J. Harvey, S.A. Kaestner, D.E. Sutter, N.G. Harvey, J.A. Mikszta and R.J. Pettis, Microneedle-based intradermal delivery enables rapid lymphatic uptake and distribution of protein drugs, Pharm. Res. 28 (2011) 107.

[5] G. Jurisic and M. Detmar, Lymphatic endothelium in health and disease, Cell Tissue Res. 335 (2009) 97.

[6] J.B. Dixon, Lymphatic lipid transport: sewer or subway?, Trends Endocrinol. Metab. 21 (2010) 480.

[7] R.F. Uren et al., Mammary Lymphoscintigraphy in Breast Cancer, J. Nucl. Med. 36 (1995) 1775.

[8] J. Norman et al., Redefinition of Cutaneous Lymphatic Drainage with the Use of Lymphoscintigraphy for Malignant-Melanoma, Am. J. Surg. 162 (1991) 432.

[9] M. Vidal et al., Accuracy and reproducibility of lymphoscintigraphy for sentinel node detection in patients with cutaneous melanoma, J. Nucl. Med. 53 (2012) 1193.

[10] D.H. Berger et al., Lymphoscintigraphy as a predictor of lymphatic drainage from cutaneous melanoma, Ann. Surg. Oncol. 4 (1997) 247. 
[11] M. Tsuchimochi and K. Hayama, Intraoperative gamma cameras for radioguided surgery: technical characteristics, performance parameters, and clinical applications, Phys. Med. 29 (2013) 126.

[12] S. Pitre, L. Menard, M. Ricard, M. Solal, J.R. Garbay and Y. Charon, A hand-held imaging probe for radio-guided surgery: physical performance and preliminary clinical experience, Eur. J. Nucl. Med. Mol. Imaging 30 (2003) 339.

[13] W.L. e2v Technologies Ltd, Chelmsford, Essex, CM1 2QU, U.K. .

[14] J.E. Lees, D.J. Bassford, O.E. Blake, P.E. Blackshaw and A.C. Perkins, A high resolution Small Field Of View (SFOV) gamma camera: a columnar scintillator coated CCD imager for medical applications, 2011 JINST 6 C12033.

[15] L.R. Ultimate Metals, Chingford, London, E4 7HZ, U.K. .

[16] S.L. Bugby, J.E. Lees, B.S. Bhatia and A.C. Perkins, Characterisation of a high resolution small field of view portable gamma camera, Phys. Med. 30 (2014) 331.

[17] C.C. Ferreira, R.E. Ximenes, C.A.B. Garcia, J.W. Vieira and A.F. Maia, Total mass attenuation coefficient evaluation of ten materials commonly used to simulate human tissue, J. Phys. Conf. Ser. 249 (2010) 012029.

[18] W.-R. Pan, C.M. le Roux and S.M. Levy, Alternative lymphatic drainage routes from the lateral heel to the inguinal lymph nodes: anatomic study and clinical implications, ANZ J. Surg. 81 (2011) 431.

[19] K. Kiyono et al., The number and size of normal mediastinal lymph nodes: a postmortem study, Am. J. Roentgenol. 150 (1988) 771.

[20] F. Giammarile et al., The EANM and SNMMI practice guideline for lymphoscintigraphy and sentinel node localization in breast cancer, Eur. J. Nucl. Med. Mol. Imaging 40 (2013) 1932.

[21] H. Suami, W.R. Pan and G.I. Taylor, Historical Review of Breast Lymphatic Studies, Clin. Anat. 22 (2009) 531

[22] W.R. Pan, H. Suami and G.I. Taylor, Senile changes in human lymph nodes, Lymphat. Res. Biol. 6 (2008) 77.

[23] M.R.S. Keshtgar and P.J. Ell, Sentinel lymph node detection and imaging, Eur. J. Nucl. Med. 26 (1999) 57.

[24] M.R. Jensen, L. Simonsen, T. Karlsmark and J. Bulow, Lymphoedema of the lower extremities-background, pathophysiology and diagnostic considerations, Clin. Physiol. Funct. Imaging 30 (2010) 389.

[25] Y. Ogawa and K. Hayashi, 99mTc-DTPA-HSA lymphoscintigraphy in lymphedema of the lower extremities: diagnostic significance of dynamic study and muscular exercise, Kaku Igaku $\mathbf{3 6}$ (1999) 31.

[26] R.A. Cambria, P. Gloviczki, J.M. Naessens and H.W. Wahner, Noninvasive evaluation of the lymphatic system with lymphoscintigraphy: a prospective, semiquantitative analysis in 386 extremities, J. Vasc. Surg. 18 (1993) 773.

[27] F.W.C. van der Ent, R.A.M. Kengen, H.A.G. van der Pol and A.G.M. Hoofwijk, Sentinel node biopsy in 70 unselected patients with breast cancer: increased feasibility by using $10 \mathrm{mCi}$ radiocolloid in combination with a blue dye tracer, Eur. J. Surg. Oncol. 25 (1999) 24.

[28] R.J. Gray, B.A. Pockaj and M.C. Roarke, Injection of (99m)Tc-labeled sulfur colloid the day before operation for breast cancer sentinel lymph node mapping is as successful as injection the day of operation, Am. J. Surg. 188 (2004) 685. 
[29] M.D. McCarter, H. Yeung, S. Yeh, J. Fey, P.I. Borgen and H.S. Cody III, Localization of the sentinel node in breast cancer: identical results with same-day and day-before isotope injection, Ann. Surg. Oncol. 8 (2001) 682.

[30] G. Mariani et al., Radioguided sentinel lymph node biopsy in malignant cutaneous melanoma, J. Nucl. Med. 43 (2002) 811.

[31] M.M.I. Systems, NUCLINE ${ }^{\mathrm{TM}}$ X-RING-R (HR), -C, Mediso Medical Imaging Systems, Hungary (2011).

[32] T. Lindeberg, Detecting salient blob-like image structures and their scales with a scale-space primal sketch: a method for focus-of-attention, Int. J. Comput. Vis. 11 (1993) 283.

[33] W.R. Tiago Ferreira, ImageJ User Guide, (2012).

[34] A. Rose, Vision: Human and Electronic, Plenum Press, New York, U.S.A. (1973).

[35] S.R. Cherry, J.A. Sorenson and M.E. Phelps, Physics in Nuclear Medicine, fourth edition, Elsevier, Philadelphia, U.S.A. (2012).

[36] D. Dickerscheid, J. Lavalaye, L. Romijn and J. Habraken, Contrast-noise-ratio (CNR) analysis and optimisation of breast-specific gamma imaging (BSGI) acquisition protocols, EJNMMI Res. 3 (2013) 21.

[37] OriginLab, User Guide, OriginLab Corporation, http://www.originlab.com/doc/.

[38] D.L. Morton et al., Technical Details of Intraoperative Lymphatic Mapping for Early Stage Melanoma, Arch. Surg. 127 (1992) 392.

[39] M.I. Ross, Role of lymphatic mapping and sentinel node biopsy in melanoma detection, Eur. J. Cancer 34 (Suppl. 3) (1998) S7.

[40] G. Mariani et al., Radioguided sentinel lymph node biopsy in patients with malignant cutaneous melanoma: the nuclear medicine contribution, J. Surg. Oncol. 85 (2004) 141. 